\title{
Nanoscale magnetic imaging of a single electron spin under ambient conditions
}

\author{
M. S. Grinolds ${ }^{1 \dagger}$, S. Hong ${ }^{2 \dagger}$, P. Maletinsky ${ }^{1,3 \dagger}$, L. Luan ${ }^{1}$, M. D. Lukin ${ }^{1}$, R. L. Walsworth ${ }^{1,4}$ and A. Yacoby ${ }^{1 \star}$
}

The detection of ensembles of spins under ambient conditions has revolutionized the biological, chemical and physical sciences through magnetic resonance imaging ${ }^{1}$ and nuclear magnetic resonance ${ }^{2,3}$. Pushing sensing capabilities to the individual-spin level would enable unprecedented applications such as single-molecule structural imaging; however, the weak magnetic fields from single spins are undetectable by conventional far-field resonance techniques ${ }^{4}$. In recent years, there has been a considerable effort to develop nanoscale scanning magnetometers ${ }^{5-8}$, which are able to measure fewer spins by bringing the sensor in close proximity to its target. The most sensitive of these magnetometers generally require low temperatures for operation, but the ability to measure under ambient conditions (standard temperature and pressure) is critical for many imaging applications, particularly in biological systems. Here we demonstrate detection and nanoscale imaging of the magnetic field from an initialized single electron spin under ambient conditions using a scanning nitrogen-vacancy magnetometer. Real-space, quantitative magnetic-field images are obtained by deterministically scanning our nitrogen-vacancy magnetometer $50 \mathrm{~nm}$ above a target electron spin, while measuring the local magnetic field using dynamically decoupled magnetometry protocols. We discuss how this single-spin detection enables the study of a variety of room-temperature phenomena in condensed-matter physics with an unprecedented combination of spatial resolution and spin sensitivity.

So far, the magnetic fields from single electron spins have been imaged only under extreme conditions (ultralow temperatures and high vacuum $)^{9}$. Magnetometers based on negatively charged nitrogen-vacancy (NV) centres in diamond have been proposed as sensors capable of measuring individual spins ${ }^{10-13}$ because they can be initialized and read-out optically ${ }^{14}$ and have long coherence times ${ }^{15}$, even under ambient conditions. Moreover, because NV centres are atomic in size, they offer significant advantages in magnetic resolution and sensing capabilities if they can be brought in close proximity of targets to be measured. Recent advances in diamond nanofabrication have allowed for the creation of robust scanning probes that host individual NV centres within roughly $25 \mathrm{~nm}$ of their tips ${ }^{16}$. Here, we employ such a scanning NV centre to image the magnetic dipole field of a single target electron spin.

Our scanning NV magnetometer (Fig. 1a) consists of a combined confocal and atomic force microscope as previously described $^{17}$, which hosts a sensing NV centre embedded in a diamond nanopillar scanning probe tip ${ }^{16}$. The sensor NV's spin state is initialized optically and read out through spin-dependent fluorescence, and its position relative to the sample is controlled through atomic-force feedback between the tip and sample. Microwaves are used to coherently manipulate the sensor NV spin. Magnetic sensing is achieved by measuring the NV spin's optically detected electron spin resonance (ESR), either by continuously applying near-resonant microwave radiation (Fig. 1b) or through pulsed spin-manipulation schemes ${ }^{12,13}$ (Fig. 1c), where the sensor $\mathrm{NV}$ spin precesses under the influence of its local magnetic field (projected along the NV centre's crystallographic orientation). We measure the contribution of the magnetic field from a target electron spin to this precession. The entire system, including both the scanning NV magnetometer and the target sample, operates under ambient conditions.

To verify the single-spin detection and imaging, we choose our target to be the spin associated with another negatively charged $\mathrm{NV}$ centre in a separate diamond crystal (so that the sensor and target $\mathrm{NV}$ centres can be scanned relative to one another). The advantage of using an NV target is that both its location and spin state can be independently determined by its optical fluorescence. As discussed below, we can thus compare the target NV's magnetically measured location to its optically measured location and ensure that the magnetic image is from a single targeted spin. Furthermore, we can guarantee that the target spin is initialized and properly modulated, as is useful for optimizing a.c. magnetic sensing.

To isolate single NV targets for imaging, NV centres are created in a shallow $(<25 \mathrm{~nm})$ layer of a bulk diamond through established implantation and annealing techniques, as used in previous work ${ }^{17}$. The target diamond surface is structured to create nanoscale mesas, whose diameters $(\sim 200 \mathrm{~nm})$ are chosen to contain, on average, a single NV spin. Mesas with single NV centres (as determined through photon auto-correlation experiments, Supplementary Fig. S1) are chosen for our measurements. To individually control the target and sensor NV spins, we choose a target NV centre with a different crystallographic orientation (which determines the spin quantization axis) from the sensor NV, so that their spin transitions can be spectrally separated in ESR measurements by applying a uniform static magnetic field.

Spatial features in the collected fluorescence from scanning the NV magnetometer over target diamond mesas allow us to independently determine the relative positions of the sensor and target NV spins (Fig. 2). First, the scanning diamond nanopillar acts as a waveguide ${ }^{18}$ that, when centred precisely above the target $\mathrm{NV}$, provides efficient collection of fluorescence from the target NV (in addition to the sensor NV). In addition, the sensor NV's fluorescence is efficiently coupled into the target bulk diamond when it is centred on a mesa, owing to the diamond's high refractive index. The intersection of these two near-field fluorescence features

${ }^{1}$ Department of Physics, Harvard University, Cambridge, Massachusetts 02138, USA, ${ }^{2}$ School of Engineering and Applied Science, Harvard University, Cambridge, Massachusetts, 02138, USA, ${ }^{3}$ Department of Physics, University of Basel, Basel, 4056 Switzerland, Switzerland, ${ }^{4}$ Harvard-Smithsonian Center for Astrophysics, Cambridge, Massachusetts 02138, USA. †These authors contributed equally to this work. *e-mail: yacoby@physics.harvard.edu. 

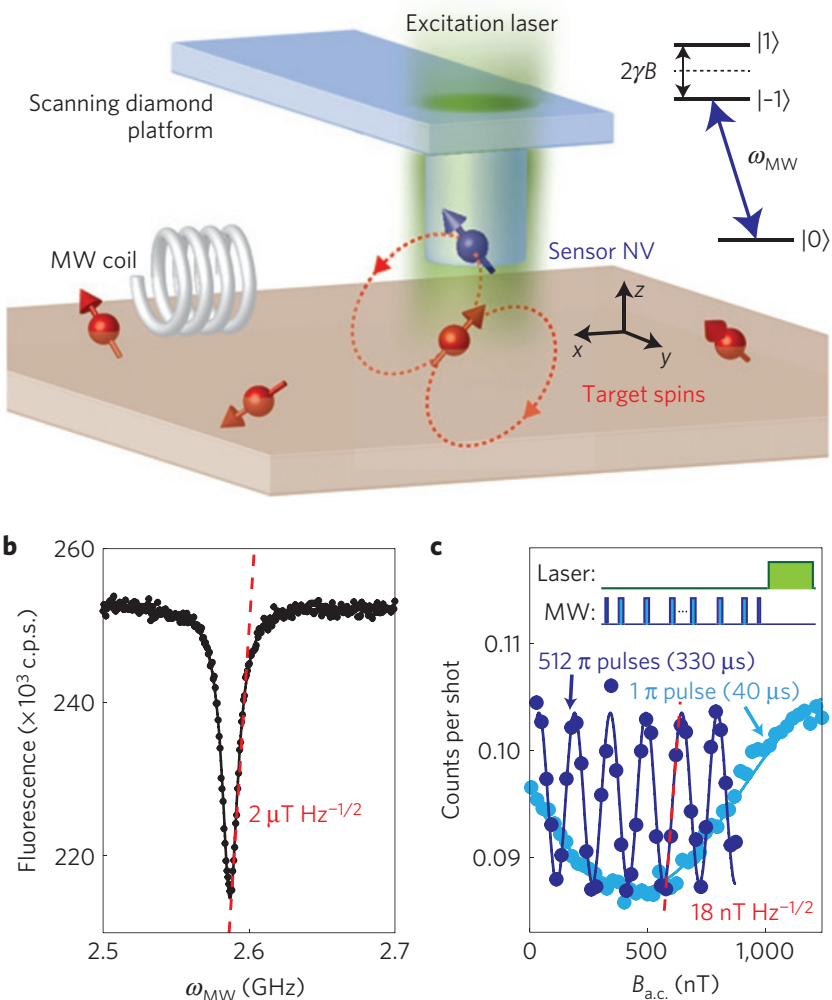

Figure 1 | Scanning NV magnetometer. a, Conceptual schematic of the scanning NV magnetometer. The sensor NV is hosted within a scanning diamond nanopillar ${ }^{16}$, where its spin is initialized and read out optically from above ( $532 \mathrm{~nm}$ excitation laser spot shown). Coherent NV spin manipulations are performed by a nearby microwave (MW) coil, in this work operating near resonance with the $|0\rangle$ to $|-1\rangle$ transition, in the presence of a static applied magnetic field (not shown). The sensor NV is scanned over target spins of interest to construct magnetic field images. b, By continuously applying the excitation and sweeping the microwaves across the $|0\rangle$ to $|-1\rangle$ transition, optically detected magnetic resonance provides a measure of the static magnetic field at the NV centre, with a d.c. sensitivity of $\sim 2 \mu \mathrm{THz}{ }^{-1 / 2}$. c, By dynamically decoupling the sensor NV spin from its environment, the sensor's magnetic field sensitivity is markedly improved for a.c. magnetic fields. Plotted are a spin-echo (1-pulse) magnetometry sequence with a $40-\mu$ s total evolution time, and a 512-pulse XY8 (ref. 21) magnetometry sequence (see Supplementary Discussion) with 330- $\mu$ s total evolution time, which achieve magnetic field sensitivities of $56 \mathrm{nT} \mathrm{Hz}^{-1 / 2}$ and $18 \mathrm{nT} \mathrm{Hz}^{-1 / 2}$, respectively.

indicates where the sensor NV spin is closest to the target NV spin. This spatial location is later used to confirm the location of the magnetically imaged target $\mathrm{NV}$ spin.

Near the expected location of the target, the local magnetic field is measured with a magnetometry pulse sequence performed on the sensor NV using a combination of dynamic decoupling ${ }^{19}$ and double electron-electron resonance ${ }^{20}$. The sensor NV spin is prepared in a superposition of spin states, where it accumulates phase proportional to the local magnetic field, including contributions from the target NV spin. To optimize magnetic field sensitivity, the sensor $\mathrm{NV}$ is dynamically decoupled from fluctuating magnetic fields in its environment (Fig. 3, upper panel) through the repeated application of microwave $\pi$-pulses. Normally, this pulse sequence would also remove any magnetic signal from a static target spin, but we also simultaneously invert the target $\mathrm{NV}$ spin in phase with the $\pi$-pulses applied to the sensor NV spin (Fig. 3, lower panel) to maintain the sign of phase accumulation by the sensor spin due to the target NV spin. The total acquired phase is converted

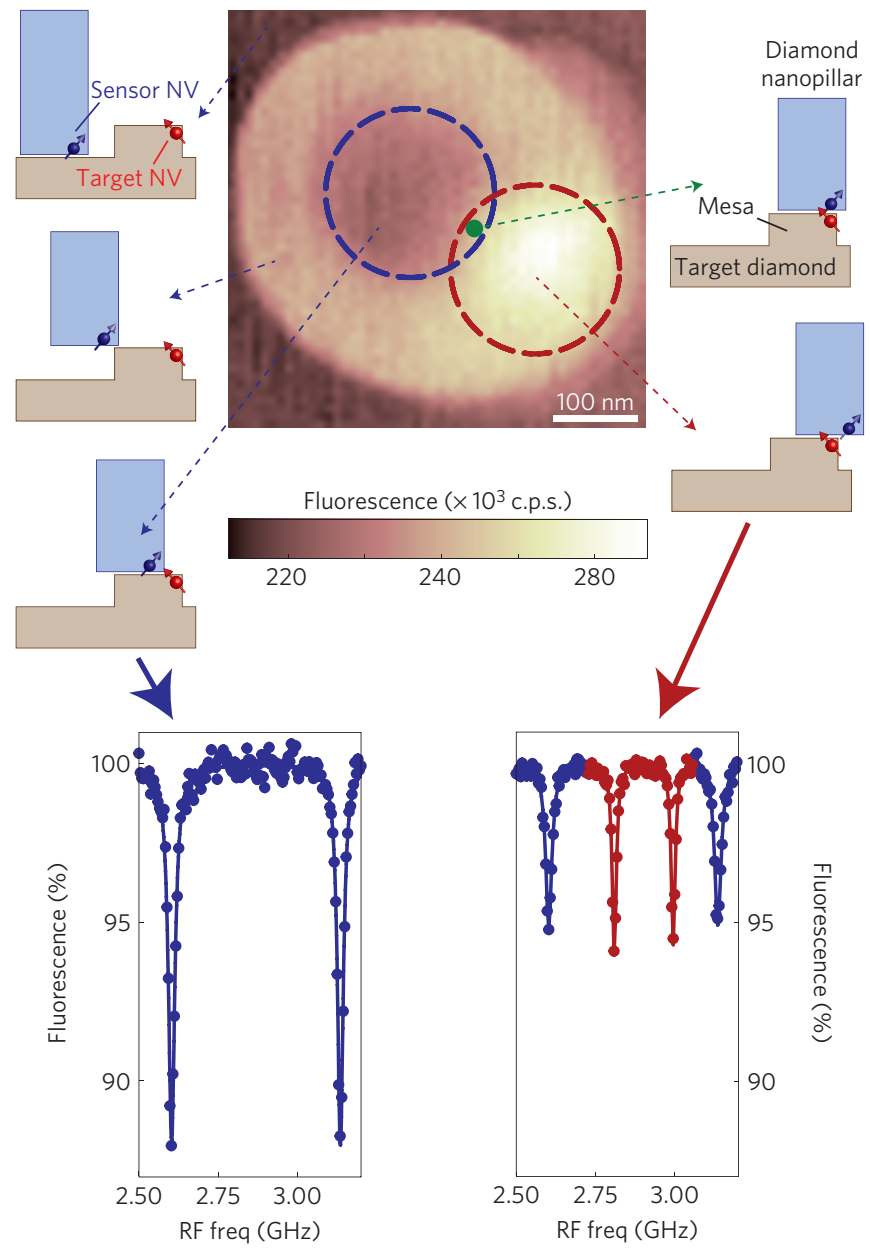

Figure 2 | Independent determination of a target spin's location. The sensor NV's diamond nanopillar is scanned over a target nanostructure ('mesa') containing a single target NV centre. The combined NV fluorescence is recorded as a function of position (top, centre panel). The fluorescence has a strong spatial dependence because sensor NV fluorescence can partially couple into the target bulk diamond when the sensor NV is close to the sample surface, and target NV fluorescence can couple into the nanopillar waveguide when the nanopillar is located above the target NV. When the nanopillar is located away from the target NV, only fluorescence from the sensor NV is collected, as indicated by ESR measurements showing two spectral peaks corresponding to the sensor $N V$ spin $m_{S}=0 / \pm 1$ transitions (bottom, left panel). For ESR measurements taken with the nanopillars located above the target NV (bottom, right panel) there are four observable spectral peaks that correspond to both the sensor and target NV spin transitions (blue and red, respectively), with reduced ESR contrast owing to collecting fluorescence from both NV spins. The centre of the target-coupling circle (red dashed circle around bright fluorescence spot) indicates the lateral location of the target NV spin relative to the centre of the nanopillar. Similarly, the centre of the sensor-quenching circle (blue dashed circle around dark fluorescence spot) indicates the lateral location of the sensor NV spin. With both NV spins' lateral locations known, the position of the sensor-target closest approach can be ascertained (green dot).

to a population difference, which is measured by means of NV spin-dependent fluorescence.

With the sensor NV in close proximity to the target diamond surface, the field sensitivity of the sensor NV is reduced, because sensor NV fluorescence is partially emitted into the target bulk diamond (owing to its high refractive index), and the target NV adds background fluorescence to magnetic measurements. Owing 

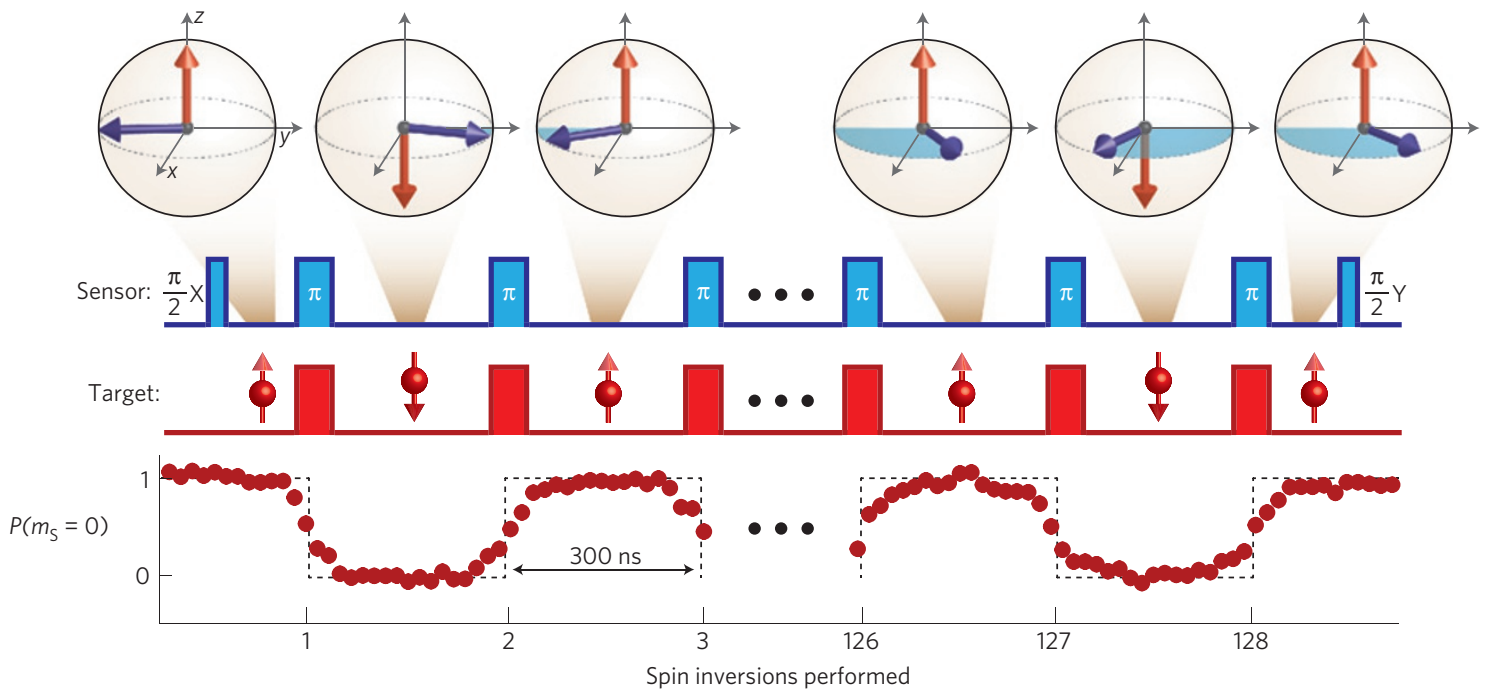

Figure 3 | Single-spin detection scheme and target spin modulation verification. To detect the magnetic field from the target NV spin, the sensor NV spin (top panel, blue arrow) is placed in a superposition of spin states with a microwave $\pi / 2$ pulse (around the $x$ axis). It then evolves under the influence of the magnetic field from the target spin (red arrow), accumulating phase (shaded blue region, whose extent is visually exaggerated for visibility). To optimize the sensor spin's magnetic sensitivity, it is dynamically decoupled from its environment (see Supplementary Discussion) ${ }^{19}$ by the repeated application of microwave $\pi$-pulses using an $X Y 8$ sequence ${ }^{21}$. To magnetically measure the target NV spin, it is inverted, synchronously with the $\pi$-pulses applied to the sensor NV, so that phase shifts induced on the sensor by the target spin constructively accumulate. (The target NV spin is modulated between the $m_{\mathrm{S}}=0$ and $m_{S}=-1$ states to isolate an effective spin-1/2 system from the target NV's spin triplet.) To invert the target NV spin with high fidelity, we employ adiabatic fast passages (see Supplementary Discussion). Plotted is the measured target NV fluorescence for pulses 1, 2, 127 and 128, indicating that the target NV spin can be modulated many times without substantial polarization decay. The sensor NV's accumulated phase is converted to a population difference using a final $\pi / 2$ pulse, whose axis $(y)$ is chosen to maximize sensitivity to small magnetic fields.

to these effects, our sensor NV's magnetic field sensitivity at the closest approach to the target $\mathrm{NV}$ is reduced to approximately $96 \mathrm{nT} \mathrm{Hz}^{-1 / 2}$ (with a 32-л-pulse XY8 (ref. 21) decoupling scheme and a $40-\mu$ s total phase accumulation time; Supplementary Fig. S5). As the target NV is embedded in bulk diamond, the sensor-to-target vertical separation is roughly twice the distance between the sensor $\mathrm{NV}$ and the diamond surface. Thus, for our magnetic field imaging of a single target $\mathrm{NV}$ spin, we expect a $\sim 50$-nm sensor-target vertical separation, which results in a magnetic field of about $10 \mathrm{nT}$ at the sensor NV location.

A magnetic field image centred at the expected target spin location is acquired by averaging the sensor's NV fluorescence in multiple scans of the NV magnetometer across a $\sim 200 \times 200-\mathrm{nm}$ field-of-view (taken using a lateral drift correction scheme detailed in Supplementary Fig. S3). A normalization scheme is applied to the magnetometry, where we alternately initialize the target $\mathrm{NV}$ spin in the $|0\rangle$ state and the $|-1\rangle$ state and measure the equal and opposite phase shifts induced during the sensor NV's magnetometry sequence (Supplementary Fig. S2). We subtract the measured NV fluorescence rates for these two initial target NV spin polarizations, which isolates the magnetic field signal from the target spin (Supplementary Fig. S4).

Near the centre of the magnetometry scan, we observe a drop in the normalized fluorescence from the magnetometry sequence that is well beyond the uncertainty set by the measurement's noise level and is consistent with the effect of a single target NV spin's magnetic field on the sensor NV. The complete magnetic field image clearly indicates the presence and location of the target NV spin (Fig. 4a). This single electron spin detection is confirmed by repeating the measurement with a spatial linecut of magnetometry measurements (Fig. 4b), with a resulting magnetic response that fits well to a vertical separation of $51.1 \pm 2.0 \mathrm{~nm}$ between the sensor and target NV centres. (Errors are determined from the $\chi^{2}$ of the fit as a function of distance, where the sensor-to-target displacement is the only free parameter and the orientations of both
NV spins are independently measured using ESR.) The measured fluorescence difference is converted to a magnetic field at the sensor NV (peak value of $8.6 \mathrm{nT}$, Fig. 4b) by using the sensor $\mathrm{NV}$ spin's independently calibrated magnetic field response and fluorescence rate. Both scanning magnetometry measurements are in good agreement with simulations of the sensor NV's response to the magnetic field from a single electron spin at a vertical distance of $51 \mathrm{~nm}$ (Fig. 4c). Thus, the above measurements are consistent and confirm the detection and nanoscale imaging of the single target spin.

In the demonstrated magnetic field imaging, single-spin measurements with a signal-to-noise ratio (SNR) of one can be acquired in $2.3 \mathrm{~min}$. The data for both single-spin measurements presented in Fig. 4 have been integrated for a total time of $42 \mathrm{~min}$ per point, yielding an SNR of 4.3, which we note is more than a 15 -fold reduction as compared with previous single-spin imaging performed at low temperatures ${ }^{9}$. This integration time is consistent with the measured target $\mathrm{NV}$ spin magnetic field $(8.6 \mathrm{nT})$ and sensor NV magnetic field sensitivity calculated by assuming the noise is dominated by photon shot noise $\left(96 \mathrm{nT} \mathrm{Hz}^{-1 / 2}\right)$. We note that the SNR for imaging individual spins increases markedly with decreasing sensor-to-target distance (Fig. 4d), because dipolar fields decay as $1 / r^{3}$, and thus only modest improvements in distance will be required to reduce data integration times to the order of seconds.

By successfully measuring the magnetic field from a single target NV spin, we have demonstrated that imaging of individual, initialized electron spins is possible under ambient conditions. At room temperature, spins are generally unpolarized; however there are numerous problems of great interest for which spins are polarized out of thermal equilibrium, and can in principle be magnetically detected. Examples include spin-polarized currents in the spin-Hall effect ${ }^{22}$ or in topological insulators ${ }^{23}$, spin-injected carriers through ferromagnetic tunnel $\operatorname{contacts}^{24}$, ferromagnetic point defects in graphene $e^{25,26}$ and optically pumped carriers and 
a

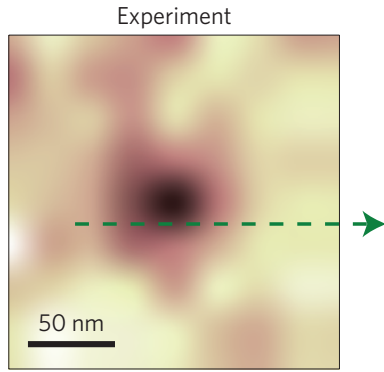

Fluorescence difference (c.p.s.)

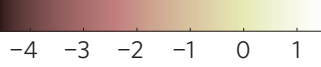

c

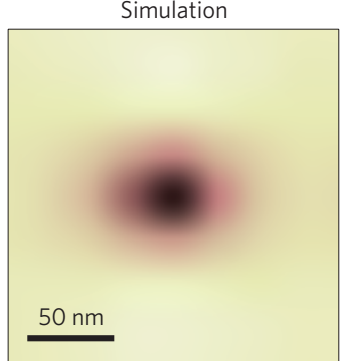

Fluorescence difference (c.p.s.)

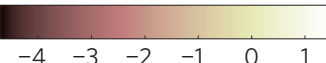

b

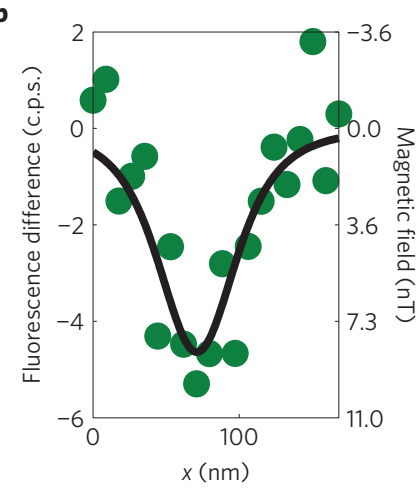

d

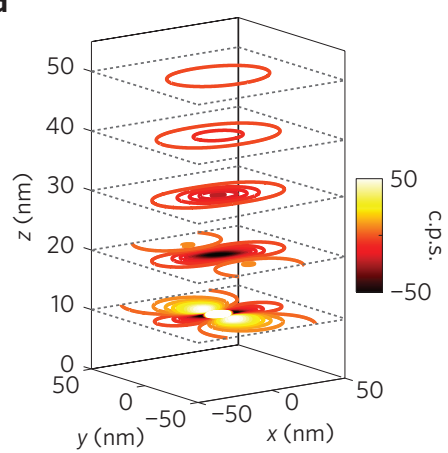

Figure 4 | Single-spin magnetic imaging. a, Magnetic field image of a target NV spin near the surface of a diamond mesa, acquired with the scanning NV magnetometer. While repeatedly running an a.c. magnetometry pulse sequence (here with a 32-pulse $X Y 8$ sequence, with $40 \mu$ s of total evolution time), the sensor NV is laterally scanned over the target, and the fluorescence rates for the target spin starting in the $|0\rangle$ state as well as the $|-1\rangle$ state are independently recorded. Plotted is the difference between these measurements, which depends only on the sensor NV's magnetic interaction with the target spin and not on background fluorescence variations (see Supplementary Information). The pronounced drop in fluorescence near the centre of the image indicates a detected single electron spin. $\mathbf{b}$, An independent magnetometry linecut taken along the green arrow confirms the single-spin imaging, which has an intensity and width consistent with the recorded image. The measured fluorescence difference is converted to the measured magnetic field using the sensor NV's calibrated field sensitivity and fluorescence rate (see Supplementary Fig. S5). c, Simulated fluorescence due to a target spin. With only the sensor-target displacement as a free parameter, the spin signal is simulated, which agrees well with both the spin image and the linecut for a vertical distance of $51 \mathrm{~nm}$ (the fit in $\mathbf{b}$ and the image in $\mathbf{c}$ have the same parameters). $\mathbf{d}$, If the sensor-target vertical distance can be moderately reduced, the quality of single-spin imaging will be markedly improved. Plotted are simulated lateral magnetic field contours from a single target electron spin for different sensor-target vertical separations where each contour indicates an increase of signal-to-noise by one for a $100 \mathrm{~s}$ integration time. At $50 \mathrm{~nm}$ (the current condition), there is only one contour, indicating single-spin imaging with a signal-to-noise of one; however, at $10 \mathrm{~nm}$, a signal-to-noise of roughly 100 is possible, such that many contours and the dipole lobes of the target spin are clearly observable. c.p.s., counts per shot.

defects $^{27}$; all of which can be (or are predicted to be) manifested at room temperature. These systems have attracted significant attention for both their underlying physics as well as their application to spintronics and quantum computation. So far such polarized spin systems have been challenging to study because their magnetic fields have been too weak to image. With single-spin sensitivity and nanoscale resolution, our magnetometer will enable magnetic measurements of these initialized spin systems.

We note that the demonstrated a.c. magnetometry techniques are generally applicable to such systems with initialized spins as various methods exist to obtain time-varying magnetic fields from a target at the sensor position. First, as was done in this work, conventional ESR can be used to flip isolated spins with long spin-relaxation times. Alternatively, many mechanisms for spin polarization can be modulated, such as currents for spin-polarized currents $^{22-24}$, gate voltages for manipulating electron density in screening-dependent ferromagnetism ${ }^{25}$, or optical polarization ${ }^{27}$. Finally, the sensor-to-target distance can be periodically varied, which allows for the conversion of static field gradients on the sample to time-varying signals at the sensor position ${ }^{28}$.

During magnetic phase accumulation, if an appreciable amount of phase can be acquired on the sensor NV from a target spin, then a single non-initialized spin could in principle also be detected sensitively by measuring its variance (Supplementary Fig. S6). If the coherent sensor-target coupling is strong enough for more than $2 \pi$ of sensor NV phase to be accumulated during magnetic field measurements, then phase-estimation techniques can be employed, thus allowing the measurement noise to decrease linearly in time $e^{29,30}$ and potentially offering a great boost in speed to magnetic imaging. Moreover, if an initialized target spin has a coherence time as long as the sensor NV, then the target and sensor spins could be entangled. Combined with long-lived storage techniques for quantum states ${ }^{31}$, the ability to entangle a scanning sensor and target spins could allow for mechanical transfer of quantum information between solid-state spins.

Received 3 September 2012; accepted 27 December 2012; published online 3 February 2013

\section{References}

1. Mansfield, P. Snapshot magnetic resonance imaging (Nobel lecture). Angew. Chem. Int. Edn Engl. 43, 5456-5464 (2004).

2. Rabi, I. I., Zacharias, J. R., Millman, S. \& Kusch, P. A new method of measuring nuclear magnetic moment. Phys. Rev. 53, 318 (1938).

3. Bloch, F. Nuclear induction. Phys. Rev. 70, 460-474 (1946).

4. Glover, P. \& Mansfield, P. Limits to magnetic resonance microscopy. Rep. Prog. Phys. 65, 1489-1511 (2002).

5. Martin, Y. \& Wickramasinghe, H. K. Magnetic imaging by 'force microscopy' with $1000 \AA$ resolution. Appl. Phys. Lett. 50, 1455-1457 (1987).

6. Chang, A. M. et al. Scanning Hall probe microscopy. Appl. Phys. Lett. 61, 1974-1976 (1992).

7. Zuger, O. \& Rugar, D. First images from a magnetic resonance force microscope. Appl. Phys. Lett. 63, 2496-2498 (1993).

8. Kirtley, J. R. et al. High-resolution scanning SQUID microscope. Appl. Phys. Lett. 66, 1138-1140 (1995).

9. Rugar, D., Budakian, R., Mamin, H. J. \& Chui, B. W. Single spin detection by magnetic resonance force microscopy. Nature 430, 329-332 (2004).

10. Balasubramanian, G. et al. Nanoscale imaging magnetometry with diamond spins under ambient conditions. Nature 455, 648-651 (2008).

11. Degen, C. L. Scanning magnetic field microscope with a diamond single-spin sensor. Appl. Phys. Lett. 92, 243111-243113 (2008).

12. Maze, J. R. et al. Nanoscale magnetic sensing with an individual electronic spin in diamond. Nature 455, 644-647 (2008).

13. Taylor, J. M. et al. High-sensitivity diamond magnetometer with nanoscale resolution. Nature Phys. 4, 810-816 (2008).

14. Gruber, A. et al. Scanning confocal optical microscopy and magnetic resonance on single defect centers. Science 276, 2012-2014 (1997).

15. Balasubramanian, G. et al. Ultralong spin coherence time in isotopically engineered diamond. Nature Mater. 8, 383-387 (2009).

16. Maletinsky, P. et al. A robust scanning diamond sensor for nanoscale imaging with single nitrogen-vacancy centres. Nature Nanotech. 7, 320-324 (2012).

17. Grinolds, M. S. et al. Quantum control of proximal spins using nanoscale magnetic resonance imaging. Nature Phys. 7, 687-692 (2011).

18. Babinec, T. M. et al. A diamond nanowire single-photon source. Nature Nanotech. 5, 195-199 (2010).

19. de Lange, G., Wang, Z. H., Ristè, D., Dobrovitski, V. V. \& Hanson, R. Universal dynamical decoupling of a single solid-state spin from a spin bath. Science 330, 60-63 (2010). 
20. Larsen, R. G. \& Singel, D. J. Double electron-electron resonance spin-echo modulation: Spectroscopic measurement of electron spin pair separations in orientationally disordered solids. J. Chem. Phys. 98, 5134-5146 (1993).

21. Gullion, T. \& Schaefer, J. Elimination of resonance offset effects in rotational-echo, double-resonance NMR. J. Magn. Reson. 92, 439-442 (1991).

22. Kimura, T., Otani, Y., Sato, T., Takahashi, S. \& Maekawa, S. Room-temperature reversible spin Hall effect. Phys. Rev. Lett. 98, 156601 (2007).

23. Zhang, $\mathrm{H}$. et al. Topological insulators in $\mathrm{Bi}_{2} \mathrm{Se}_{3}, \mathrm{Bi}_{2} \mathrm{Te}_{3}$ and $\mathrm{Sb}_{2} \mathrm{Te}_{3}$ with a single Dirac cone on the surface. Nature Phys. 5, 438-442 (2009).

24. Dash, S. P., Sharma, S., Patel, R. S., de Jong, M. P. \& Jansen, R. Electrical creation of spin polarization in silicon at room temperature. Nature 462, 491-494 (2009).

25. Pisani, L., Montanari, B. \& Harrison, N. M. A defective graphene phase predicted to be a room temperature ferromagnetic semiconductor. New J. Phys. 10, 033002 (2008).

26. Santos, E. J. G., Ayuela, A. \& Sánchez-Portal, D. Universal magnetic properties of $\mathrm{sp}^{3}$-type defects in covalently functionalized graphene. New J. Phys. 14, 043022 (2012)

27. Wang, X. J. et al. Room temperature defect-engineered spin filter based on non-magnetic semiconductor. Nature Mater. 8, 198-202 (2009).

28. Hong, S. et al. Coherent, mechanical control of a single electronic spin. Nano Lett. 12, 3920-3924 (2012).

29. Waldherr, G. et al. High-dynamic-range magnetometry with a single nuclear spin in diamond. Nature Nanotech. 7, 105-108 (2012).
30. Nusran, N. M., Momeen, M. U. \& Dutt, M. V. G. High-dynamic-range magnetometry with a single electronic spin in diamond. Nature Nanotech. 7, 109-113 (2012).

31. Maurer, P. C. et al. Room-temperature quantum bit memory exceeding one second. Science 336, 1283-1286 (2012).

\section{Acknowledgements}

We gratefully acknowledge Element Six, for providing diamond samples for our NV sensors and targets. M.S.G. is supported through fellowships from the Department of Defense (NDSEG programme) and the National Science Foundation. S.H. acknowledges support from the Kwanjeong Scholarship Foundation, and P.M. thanks the Swiss National Science Foundation for fellowship funding. This work was supported by the DARPA QuEST and QuASAR programmes and the MURI QuISM.

\section{Author contributions}

All authors contributed to all aspects of this work.

\section{Additional information}

Supplementary information is available in the online version of the paper. Reprints and permissions information is available online at www.nature.com/reprints.

Correspondence and requests for materials should be addressed to A.Y.

\section{Competing financial interests}

The authors declare no competing financial interests. 